ANNALES

POLONICI MATHEMATICI

$92.3(2007)$

\title{
On strong tracts of subharmonic functions of infinite lower order
}

\author{
by I. I. Marchenko and A. Szkibiel (Szczecin)
}

\begin{abstract}
The notion of a strong asymptotic tract for subharmonic functions is defined. Eremenko's value $b(\infty, u)$ for subharmonic functions is introduced and it is used to provide an exact upper estimate of the number of strong tracts of subharmonic functions of infinite lower order. It is also shown that $b(\infty, u) \leq \pi$ for subharmonic functions of infinite lower order.
\end{abstract}

1. Introduction. Let $u(z)$ be a function subharmonic in the plane and with infinite lower order, which means that

$$
\liminf _{r \rightarrow \infty} \frac{\log \max \{u(z):|z|=r\}}{\log r}=\infty .
$$

Let us consider the sets $\mathrm{E}(n)=\{z \in \mathbb{C}: u(z) \geq n\}$ for $n \in \mathbb{N}$. Let $\mathrm{C}(n)$ be a thick component of $\mathrm{E}(n)$, which means that $u(z) \not \equiv n$ on $\mathrm{C}(n)$ (see [7]). There is $k \in \mathbb{N}$ such that for every $n \geq k$ the function $u(z)$ is unbounded on all thick components $\mathrm{C}(n)$. We call a thick component $\mathrm{C}(n)$ an $n$-tract of $u(z)$. Let $n_{2}>n_{1}>k$. Then every $n_{1}$-tract contains at least one $n_{2}$-tract. If $\mathrm{q}(n)$ is the number of different $n$-tracts $\mathrm{C}(n)$, then $\mathrm{q}\left(n_{2}\right) \geq \mathrm{q}\left(n_{1}\right)$. We call $q=\lim _{n \rightarrow \infty} \mathrm{q}(n)$ the number of tracts of the function $u(z)$.

If $\mathrm{C}(n)$ is an $n$-tract of $u(z)$, then (see [11]) there exists a continuous curve $\Gamma \subset \mathrm{C}(n)$ given by the equation $z=z(t)$, where $0 \leq t<\infty$ and $z(t) \rightarrow \infty$ as $t \rightarrow \infty$, such that

$$
\lim _{\substack{z \rightarrow \infty \\ z \in \Gamma}} u(z)=\lim _{t \rightarrow \infty} u(z(t))=\infty .
$$

TheOREM A ([7]). Every subharmonic function in the plane has at least one tract.

Definition. We call an $n$-tract $\mathrm{C}(n)$ of a subharmonic function $u(z)$ a strong $n$-tract if there is a continuous curve $\Gamma \subset \mathrm{C}(n), z=z(t), 0 \leq t<\infty$,

2000 Mathematics Subject Classification: Primary 31A05; Secondary 30D20.

Key words and phrases: subharmonic function, strong tract. 
such that $z(t) \rightarrow \infty$ as $t \rightarrow \infty$ and

$$
\lim _{t \rightarrow \infty} \frac{u(z(t))}{\max \{u(z):|z|=|z(t)|\}}=1 .
$$

Let $\mathrm{p}(n)$ be the number of strong $n$-tracts of the function $u(z)$. We call $p=\lim _{n \rightarrow \infty} \mathrm{p}(n)$ the number of strong tracts of the function $u(z)$.

In [10] we provided a similar definition of strong tracts for subharmonic functions of finite lower order and estimated, in that case, the number of strong tracts.

In this paper we are going to estimate the number of strong tracts of subharmonic functions of infinite lower order. To state our results, we introduce Eremenko's value for a subharmonic function $u(z)$ (see [3]) as

$$
b(\infty, u)=\liminf _{r \rightarrow \infty} \frac{\max \{u(z):|z|=r\}}{r T_{-}^{\prime}(r, u)},
$$

where $T(r, u)$ is the Nevanlinna characteristic of $u(z)$ and $T_{-}^{\prime}(r, u)$ is its left derivative (see [7]).

We prove the following results.

THEOREM 1. For a subharmonic function u(z) of infinite lower order we have

$$
b(\infty, u) \leq \pi .
$$

This result was obtained for $u(z)=\log |f(z)|$, where $f(z)$ is a meromorphic function, by Bergweiler and Bock in [2]. The estimate given in Theorem 1 is exact (see [6]).

THEOREM 2. Let $u(z)$ be a subharmonic function with infinite lower order and with $p$ different strong tracts. Then

$$
p \leq\left[\frac{\pi}{b(\infty, u)}\right] .
$$

In the case $u(z)=\log |f(z)|$, where $f(z)$ is an entire function, the theorem was proved by one of the authors ([9]). The estimate of Theorem 2 is exact (see [9]).

CoROllary. If $u(z)$ is a subharmonic function satisfying $b(\infty, u)>0$, then the number of different strong tracts of $u(z)$ is finite.

The condition $b(\infty, u)>0$ is essential for the Corollary, because for the function $u(z)=\log \left|e^{e^{z}}\right|, b(\infty, u)=0$ and $u(z)$ has infinitely many strong tracts.

2. Auxiliary results. Let $u(z)$ be a subharmonic function. Let

$$
m^{*}(r, \theta, u)=\frac{1}{2 \pi} \sup _{|E|=2 \theta} \int_{E} u^{+}\left(r e^{i \varphi}\right) d \varphi,
$$


where $|E|$ is the Lebesgue measure of the set $E$ and $u^{+}(z)=\max \{u(z), 0\}$, be the function defined by Baernstein (see [1]). According to Baernstein's theorem the function $m^{*}(r, \theta, u)$ is subharmonic in $K=\left\{r e^{i \theta}: 0<r<\infty\right.$, $0<\theta<\pi\}$, continuous on $K \cup(\mathbb{R} \backslash\{0\}) \times\{0\}$ and convex in $\log r$ for any fixed $\theta \in[0, \pi]$. Also the function defined above is nondecreasing with respect to $r$ for any fixed $\theta$. Moreover

$$
m^{*}(r, \theta, u)=\frac{1}{\pi} \int_{0}^{\theta} \widetilde{u}\left(r e^{i \varphi}\right) d \varphi, \quad \frac{\partial m^{*}}{\partial \theta}(r, \theta, u)=\frac{1}{\pi} \widetilde{u}\left(r e^{i \theta}\right), \quad 0<\theta<\pi,
$$

where $\widetilde{u}\left(r e^{i \theta}\right)$ is the circular rearrangement of the function $u^{+}\left(r e^{i \theta}\right)([5])$.

Every subharmonic function $u(z)$ is a pointwise limit of a nonincreasing sequence $\left\{v^{k}(z)\right\}$ of subharmonic functions with continuous partial derivatives of second order $([7])$.

Lemma A ([10]). The sequence $\left\{m^{*}\left(r, \theta, v^{k}\right)\right\}$ converges to $m^{*}(r, \theta, u)$ uniformly on the set $\left\{r e^{i \theta}: 1 \leq r \leq R, 0 \leq \theta \leq \pi\right\}$ for every $R>1$.

For a real function $\varphi(r)$, we consider the operator

$$
\mathrm{L} \varphi(r)=\liminf _{h \rightarrow 0} \frac{\varphi\left(r e^{h}\right)+\varphi\left(r e^{-h}\right)-2 \varphi(r)}{h^{2}} .
$$

If the function $\varphi(r)$ is twice differentiable in $r$, then

$$
\mathrm{L} \varphi(r)=r \frac{d}{d r} r \frac{d}{d r} \varphi(r) .
$$

Since $m^{*}\left(r, \theta, u_{j}\right)$ is convex in $\log r$, for all $r>0$ and $\theta \in[0, \pi]$ we have

$$
\mathrm{Lm}^{*}\left(r, \theta, u_{j}\right) \geq 0 .
$$

Lemma B ([10,9]). Let $u(z)$ be a subharmonic function with continuous partial derivatives of second order. For all $r>0$ and for almost all $\theta \in(0, \pi)$ we have

$$
\mathrm{L}^{*}\left(r, \theta, u_{j}\right) \geq-\left.\frac{1}{\pi} \frac{\partial \widetilde{u_{j}}\left(r e^{i \varphi}\right)}{\partial \varphi}\right|_{\varphi=\theta} .
$$

Bergweiler and Bock introduced in [2] the sequences of Pólya peaks for meromorphic functions of infinite lower order. Such sequences can also be constructed for subharmonic functions, so let us recall the basic facts. For any sequences $M_{n} \rightarrow \infty$ and $\varepsilon_{n} \rightarrow 0$ there are sequences $\varrho_{n} \rightarrow \infty$ and $\mu_{n} \rightarrow \infty$ such that, for every $r$ satisfying $\log \left(r / \varrho_{n}\right) \leq M_{n} / \mu_{n}$, we have

$$
T(r, u) \leq\left(1+\varepsilon_{n}\right)\left(\frac{r}{\varrho_{n}}\right)^{\mu_{n}} T\left(\varrho_{n}, u\right) .
$$


We put $P_{n}=\varrho_{n} e^{-M_{n} / \mu_{n}}$ and $Q_{n}=\varrho_{n} e^{M_{n} / \mu_{n}}$. Then inequality (1) holds for all $r \in\left[P_{n}, Q_{n}\right]$. We assume that $M_{n}>1$. Consider the sets

$$
\begin{aligned}
& A_{n}=\left\{r \in\left[\varrho_{n}, Q_{n}\right]: T(r, u) \leq \frac{1}{\sqrt{M_{n}}}\left(\frac{r}{\varrho_{n}}\right)^{\mu_{n}} T\left(\varrho_{n}, u\right)\right\}, \\
& B_{n}=\left\{r \in\left[P_{n}, \varrho_{n}\right]: T(r, u) \leq \frac{1}{\sqrt{M_{n}}}\left(\frac{r}{\varrho_{n}}\right)^{\mu_{n}} T\left(\varrho_{n}, u\right)\right\} .
\end{aligned}
$$

We put

$$
\begin{gathered}
R_{n}=\left\{\begin{array}{ll}
\min A_{n} & \text { if } A_{n} \neq \emptyset, \\
Q_{n} & \text { if } A_{n}=\emptyset,
\end{array} \quad t_{n}= \begin{cases}\max B_{n} & \text { if } B_{n} \neq \emptyset, \\
P_{n} & \text { if } B_{n}=\emptyset,\end{cases} \right. \\
T_{n}=e^{-2 / \mu_{n}} R_{n} .
\end{gathered}
$$

Then $t_{n}<\varrho_{n}<T_{n}<R_{n}$. We may achieve that $M_{n} \rightarrow \infty$ as slowly as we please, so we have the following lemma.

Lemma C ([2]). For a subharmonic function of infinite lower order and the sequences defined above,

$$
\frac{T\left(R_{n}, u\right)}{R_{n}^{\mu_{n}}}+\frac{T\left(T_{n}, u\right)}{T_{n}^{\mu_{n}}}+\frac{T\left(t_{n}, u\right)}{t_{n}^{\mu_{n}}}=o\left(\mu_{n} \int_{t_{n}}^{T_{n}} \frac{T(r, u)}{r^{\mu_{n}+1}} d r\right), \quad n \rightarrow \infty .
$$

We need one more lemma.

Lemma D ([8]). Let a function $f(x)$ be nondecreasing on an interval $[a, b]$ and let $\varphi(x)$ be a nonnegative function having a bounded derivative on $[a, b]$. Then

$$
\int_{a}^{b} f^{\prime}(x) \varphi(x) d x \leq\left. f(x) \varphi(x)\right|_{a} ^{b}-\int_{a}^{b} \varphi^{\prime}(x) f(x) d x .
$$

Now let $\left\{v^{k}(z)\right\}$ be a nonincreasing sequence of subharmonic functions with continuous partial derivatives of second order, pointwise converging to $u(z)$. For every $k$ and for every $n$ we put (see [4])

$$
\sigma_{k, n}(r)=\int_{0}^{\pi / 2 \mu_{n}} m^{*}\left(r, \theta, v^{k}\right) \cos \mu_{n} \theta d \theta
$$

LEMMA 1. For every $\varepsilon>0$ and sequences $\left\{\mu_{n}\right\}$ and $\left\{T_{n}\right\}$ defined above, there exist $N(\varepsilon) \in \mathbb{N}$ and $K(\varepsilon, n) \in \mathbb{N}$ such that

$$
\frac{\left(\sigma_{k, n}\right)_{-}^{\prime}\left(T_{n}\right)}{T_{n}^{\mu_{n}-1}}+\frac{\mu_{n} \sigma_{k, n}\left(T_{n}\right)}{T_{n}^{\mu_{n}}}<\varepsilon \mu_{n} \int_{t_{n}}^{T_{n}} \frac{T(r, u)}{r^{\mu_{n}+1}} d r
$$

for $n>N(\varepsilon)$ and $k>K(\varepsilon, n)$. 
Proof. Let $\varepsilon>0$ be given. Let $\left\{T_{n}\right\},\left\{R_{n}\right\}$ and $\left\{\mu_{n}\right\}$ be the sequences defined above. By Lemma $\mathrm{C}$ there exists $N \in \mathbb{N}$ such that for $n>N$,

$$
\frac{T\left(R_{n}, u\right)}{R_{n}^{\mu_{n}}}+\frac{T\left(T_{n}, u\right)}{T_{n}^{\mu_{n}}}<\frac{\varepsilon}{8} \mu_{n} \int_{t_{n}}^{T_{n}} \frac{T(r, u)}{r^{\mu_{n}+1}} d r .
$$

Let us choose $N(\varepsilon) \geq N$ so that

$$
\varepsilon<T\left(T_{n}, u\right) .
$$

Applying Lemma A, for $k>K(\varepsilon, n)$, we get

$$
m^{*}\left(r, \theta, v^{k}\right) \leq m^{*}(r, \theta, u)+\varepsilon \leq T(r, u)+\varepsilon
$$

on the set $\left\{r e^{i \theta}: 1 \leq r \leq R_{n}, 0 \leq \theta \leq \pi\right\}$. Hence

$$
\begin{aligned}
\sigma_{k, n}(r) & =\int_{0}^{\pi / 2 \mu_{n}} m^{*}\left(r, \theta, v^{k}\right) \cos \mu_{n} \theta d \theta \leq \int_{0}^{\pi / 2 \mu_{n}}(T(r, u)+\varepsilon) \cos \mu_{n} \theta d \theta \\
& =\frac{1}{\mu_{n}}(T(r, u)+\varepsilon) .
\end{aligned}
$$

Therefore

$$
\frac{\mu_{n} \sigma_{k, n}\left(T_{n}\right)}{T_{n}^{\mu_{n}}} \leq \frac{T\left(T_{n}, u\right)+\varepsilon}{T_{n}^{\mu_{n}}} \leq \frac{2 T\left(T_{n}, u\right)}{T_{n}^{\mu_{n}}} .
$$

Now, because $m^{*}\left(r, \theta, v^{k}\right)$ is a nondecreasing function of $r$,

$$
\left(\sigma_{k, n}\right)_{-}^{\prime}(r)=\int_{0}^{\pi / 2 \mu_{n}} \frac{\partial m^{*}\left(r, \theta, v^{k}\right)}{\partial r} \cos \mu_{n} \theta d \theta
$$

Since $m^{*}\left(r, \theta, v^{k}\right)$ is convex in $\log r$ if $\theta$ is fixed, $r \partial m^{*}\left(r, \theta, v^{k}\right) / \partial r$ is nondecreasing and from Lemma $\mathrm{D}$ for $x>r$, we get

$$
\begin{aligned}
m^{*}\left(x, \theta, v^{k}\right) & \geq m^{*}\left(x, \theta, v^{k}\right)-m^{*}\left(r, \theta, v^{k}\right) \geq \int_{r}^{x} \frac{\partial m^{*}\left(t, \theta, v^{k}\right)}{\partial t} d t \\
& =\int_{r}^{x} \frac{t \frac{\partial}{\partial t} m^{*}\left(t, \theta, v^{k}\right)}{t} d t=\int_{r}^{x} t \frac{\partial m^{*}\left(t, \theta, v^{k}\right)}{\partial t} d(\log t) \\
& \geq r \frac{\partial m^{*}\left(r, \theta, v^{k}\right)}{\partial r} \log \frac{x}{r} .
\end{aligned}
$$

Hence

$$
\frac{\partial m^{*}\left(r, \theta, v^{k}\right)}{\partial r} \leq \frac{1}{r \log \frac{x}{r}} m^{*}\left(x, \theta, v^{k}\right) \leq \frac{1}{r \log \frac{x}{r}}(T(x, u)+\varepsilon),
$$

so, using $T_{n}=e^{-2 / \mu_{n}} R_{n}$, we have

$$
\left.\frac{\partial m^{*}\left(r, \theta, v^{k}\right)}{\partial r}\right|_{r=T_{n}} \leq \frac{1}{T_{n} \log \frac{R_{n}}{T_{n}}}\left(T\left(R_{n}, u\right)+\varepsilon\right) \leq \frac{\mu_{n}}{T_{n}} T\left(R_{n}, u\right) .
$$


Thus

$$
\begin{aligned}
\frac{\left(\sigma_{k, n}\right)_{-}^{\prime}\left(T_{n}\right)}{T_{n}^{\mu_{n}-1}} & =\left.\frac{1}{T_{n}^{\mu_{n}-1}} \int_{0}^{\pi / 2 \mu_{n}} \frac{\partial m^{*}\left(r, \theta, v^{k}\right)}{\partial r}\right|_{r=T_{n}} \cos \mu_{n} \theta d \theta \\
& \leq \frac{1}{T_{n}^{\mu_{n}}} T\left(R_{n}, u\right)=\frac{e^{2} T\left(R_{n}, u\right)}{R_{n}^{\mu_{n}}}<8 \frac{T\left(R_{n}, u\right)}{R_{n}^{\mu_{n}}}
\end{aligned}
$$

Lemma 1 follows from (3), (4) and (5).

3. Proof of Theorem 1. Applying the definition of $\sigma_{k, n}(r)$ and Lemma B, we get

$$
\mathrm{L} \sigma_{k, n}(r) \geq-\frac{1}{\pi} \int_{0}^{\pi / 2 \mu_{n}} \frac{\partial \widetilde{v^{k}}\left(r e^{i \theta}\right)}{\partial \theta} \cos \mu_{n} \theta d \theta .
$$

Integrating by parts we obtain

$$
\text { (1) } \begin{aligned}
\mathrm{L} \sigma_{k, n}(r) & \geq-\left.\frac{1}{\pi} \widetilde{v^{k}}\left(r e^{i \theta}\right) \cos \mu_{n} \theta\right|_{0} ^{\pi / 2 \mu_{n}}-\frac{\mu_{n}}{\pi} \int_{0}^{\pi / 2 \mu_{n}} \widetilde{v^{k}}\left(r e^{i \theta}\right) \sin \mu_{n} \theta d \theta \\
& =\frac{1}{\pi} \widetilde{v^{k}}(r)-\mu_{n} m^{*}\left(r, \frac{\pi}{2 \mu_{n}}, v^{k}\right)+\mu_{n}^{2} \int_{0}^{\pi / 2 \mu_{n}} m^{*}\left(r, \theta, v^{k}\right) \cos \mu_{n} \theta d \theta \\
& =\frac{1}{\pi} \widetilde{v^{k}}(r)-\mu_{n} m^{*}\left(r, \frac{\pi}{2 \mu_{n}}, v^{k}\right)+\mu_{n}^{2} \sigma_{k, n}(r) \\
& \equiv h_{k, n}(r)+\mu_{n}^{2} \sigma_{k, n}(r) .
\end{aligned}
$$

Since $\mathrm{Lm}^{*}\left(r, \theta, v^{k}\right) \geq 0$, from Fatou's lemma we get

$$
\mathrm{L} \sigma_{k, n}(r) \geq \int_{0}^{\pi / 2 \mu_{n}} \operatorname{Lm}^{*}\left(r, \theta, v^{k}\right) \cos \mu_{n} \theta d \theta \geq 0
$$

and $\sigma_{k, n}(r)$ is convex in $\log r$. It follows that $r\left(\sigma_{k, n}\right)_{-}^{\prime}(r)$ is an increasing function on $(0, \infty)$. Thus, for almost all $r>0$ we have

$$
\mathrm{L} \sigma_{k, n}(r)=r \frac{d}{d r} r\left(\sigma_{k, n}\right)_{-}^{\prime}(r) .
$$

We now divide the inequality (1) by $r^{\mu_{n}+1}$ and integrate it over the interval $\left[t_{n}, T_{n}\right]$ to obtain

$$
\int_{t_{n}}^{T_{n}} \frac{\frac{d}{d r} r\left(\sigma_{k, n}\right)_{-}^{\prime}(r)}{r^{\mu_{n}}} d r \geq \int_{t_{n}}^{T_{n}} \frac{h_{k, n}(r)}{r^{\mu_{n}+1}} d r+\mu_{n}^{2} \int_{t_{n}}^{T_{n}} \frac{\sigma_{k, n}(r)}{r^{\mu_{n}+1}} d r
$$

where the numbers $t_{n}$ and $T_{n}$ are defined just before Lemma C. Since 
the function $r\left(\sigma_{k, n}\right)_{-}^{\prime}(r)$ is increasing, by Lemma A we have

$$
\int_{t_{n}}^{T_{n}} \frac{\frac{d}{d r} r\left(\sigma_{k, n}\right)_{-}^{\prime}(r)}{r^{\mu_{n}}} d r \leq\left.\left(\frac{r\left(\sigma_{k, n}\right)_{-}^{\prime}(r)}{r^{\mu_{n}}}+\mu_{n} \frac{\sigma_{k, n}(r)}{r^{\mu_{n}}}\right)\right|_{t_{n}} ^{T_{n}}+\mu_{n}^{2} \int_{t_{n}}^{T_{n}} \frac{\sigma_{k, n}(r)}{r^{\mu_{n}+1}} d r .
$$

Hence

$$
\int_{t_{n}}^{T_{n}} \frac{h_{k, n}(r)}{r^{\mu_{n}+1}} d r \leq\left.\left(\frac{\left(\sigma_{k, n}\right)_{-}^{\prime}(r)}{r^{\mu_{n}-1}}+\mu_{n} \frac{\sigma_{k, n}(r)}{r^{\mu_{n}}}\right)\right|_{t_{n}} ^{T_{n}} .
$$

Let $\varepsilon>0$ be given. By Lemma 1, for $n>N(\varepsilon)$ and $k>K(\varepsilon, n)$, we have

$$
\int_{t_{n}}^{T_{n}} \frac{h_{k, n}(r)}{r^{\mu_{n}+1}} d r<\varepsilon \mu_{n} \int_{t_{n}}^{T_{n}} \frac{T(r, u)}{r^{\mu_{n}+1}} d r
$$

Now, if $\left\{v^{k}(z)\right\}$ is a nonincreasing sequence tending to $u(z)$, then $\left\{\widetilde{v^{k}}(z)\right\}$ is a nonincreasing sequence tending to $\widetilde{u}(z)$. By Lemma A, $m^{*}\left(r, \pi / 2 \mu_{n}, v^{k}\right)$ converges to $m^{*}\left(r, \pi / 2 \mu_{n}, u\right)$. Hence, using the monotone convergence theorem, we get

$$
\int_{t_{n}}^{T_{n}} \frac{h_{n}(r)}{r^{\mu_{n}+1}} d r<\varepsilon \mu_{n} \int_{t_{n}}^{T_{n}} \frac{T(r, u)}{r^{\mu_{n}+1}} d r
$$

where

$$
h_{n}(r)=\frac{1}{\pi} \widetilde{u}(r)-\mu_{n} m^{*}\left(r, \frac{\pi}{2 \mu_{n}}, u\right) \geq \frac{1}{\pi} \widetilde{u}(r)-\mu_{n} T(r, u) .
$$

Applying Lemma C, we have

$$
\begin{aligned}
\mu_{n} \int_{t_{n}}^{T_{n}} \frac{T(r, u)}{r^{\mu_{n}+1}} d r & =-\left.\frac{T(r, u)}{r^{\mu_{n}}}\right|_{t_{n}} ^{T_{n}}+\int_{t_{n}}^{T_{n}} \frac{r T_{-}^{\prime}(r, u)}{r^{\mu_{n}+1}} d r \\
& \leq o\left(\mu_{n} \int_{t_{n}}^{T_{n}} \frac{T(r, u)}{r^{\mu_{n}+1}} d r\right)+\int_{t_{n}}^{T_{n}} \frac{r T_{-}^{\prime}(r, u)}{r^{\mu_{n}+1}} d r \\
& \leq \frac{1}{1-\varepsilon} \int_{t_{n}}^{T_{n}} \frac{r T_{-}^{\prime}(r, u)}{r^{\mu_{n}+1}} d r .
\end{aligned}
$$

Now, using (2), (3) and (4) we get

$$
\int_{t_{n}}^{T_{n}} \frac{\widetilde{u}(r)}{r^{\mu_{n}+1}} d r<\pi \frac{1+\varepsilon}{1-\varepsilon} \int_{t_{n}}^{T_{n}} \frac{r T_{-}^{\prime}(r, u)}{r^{\mu_{n}+1}} d r .
$$

Hence there is a sequence $r_{n} \in\left[t_{n}, T_{n}\right]$ such that

$$
\widetilde{u}\left(r_{n}\right)<\pi \frac{1+\varepsilon}{1-\varepsilon} r_{n} T_{-}^{\prime}\left(r_{n}, u\right)
$$


for large enough $n$ 's. Since $\widetilde{u}\left(r_{n}\right) \geq \max \left\{u(z):|z|=r_{n}\right\}$, using the definition of $b(\infty, u)$, we get

$$
\widetilde{u}\left(r_{n}\right)>(1-\varepsilon) b(\infty, u) r_{n} T_{-}^{\prime}\left(r_{n}, u\right) .
$$

Therefore

$$
(1-\varepsilon) b(\infty, u)<\pi \frac{1+\varepsilon}{1-\varepsilon},
$$

or

$$
b(\infty, u)<\pi \frac{1+\varepsilon}{(1-\varepsilon)^{2}} .
$$

Since $\varepsilon>0$ is arbitrary we obtain Theorem 1 .

4. Proof of Theorem 2. Let $u(z)$ be a subharmonic function and let $p$ be the number of strong tracts of $u(z)$. Let $q \leq p$ be a natural number. Then there exists $n_{0} \in \mathbb{N}$ such that for all $n \geq n_{0}, \mathrm{E}(n)$ has at least $q$ connected components. Consider the functions (see [7])

$$
u_{j}(z)= \begin{cases}u(z) & \text { if } z \in C_{j}\left(n_{0}\right), \\ n_{0} & \text { if } z \notin C_{j}\left(n_{0}\right),\end{cases}
$$

for $j=1, \ldots, q$. The functions $u_{j}(z)$ are subharmonic in $\mathbb{C}$. Also, for every $j \in\{1, \ldots, q\}, u_{j}(z)$ is the pointwise limit of a nonincreasing sequence $\left\{v_{j}^{k}(z)\right\}$ of subharmonic functions with continuous partial derivatives of second order.

Now, we define the function

$$
m_{0}^{*}(r, \theta, u)=\sum_{j=1}^{q} m^{*}\left(r, \theta, u_{j}\right) .
$$

The function $m_{0}^{*}(r, \theta, u)$, being the sum of $m^{*}\left(r, \theta, u_{j}\right)$ for $j \in\{1, \ldots, q\}$, is subharmonic in $K=\left\{r e^{i \theta}: 0<r<\infty, 0<\theta<\pi\right\}$, continuous on $K \cup(\mathbb{R} \backslash\{0\}) \times\{0\}$ and convex in $\log r$ for any fixed $\theta \in[0, \pi]$. It is also nondecreasing with respect to $r$ for any fixed $\theta$.

For every $k$ and for every $n$ we put

$$
\sigma_{k, n}(r)=\int_{0}^{\pi / 2 \mu_{n}}\left(m_{k}\right)_{0}^{*}(r, \theta) \cos \mu_{n} \theta d \theta
$$

where $\left(m_{k}\right)_{0}^{*}(r, \theta)=\sum_{j=1}^{q} m^{*}\left(r, \theta, v_{j}^{k}\right)$.

It is easy to see that Lemma 1 also holds for $\sigma_{k, n}(r)$ defined above.

Now, using the definition of $\sigma_{k, n}(r)$ and Lemma B, we have

$$
\mathrm{L} \sigma_{k, n}(r) \geq-\frac{1}{\pi} \sum_{j=1}^{q} \int_{0}^{\pi / 2 \mu_{n}} \frac{\partial \widetilde{v_{j}^{k}}\left(r e^{i \theta}\right)}{\partial \theta} \cos \mu_{n} \theta d \theta .
$$


Proceeding analogously to the proof of Theorem 1 we get

$$
h_{k, n}(r)=\frac{1}{\pi} \sum_{j=1}^{q} \widetilde{v_{j}^{k}}(r)-\mu_{n}\left(m_{k}\right)_{0}^{*}\left(r, \frac{\pi}{2 \mu_{n}}\right)
$$

and

$$
h_{n}(r)=\frac{1}{\pi} \sum_{j=1}^{q} \widetilde{u}_{j}(r)-\mu_{n} m_{0}^{*}\left(r, \frac{\pi}{2 \mu_{n}}, u\right) .
$$

Then

$$
h_{n}(r) \geq \frac{1}{\pi} \sum_{j=1}^{q} \widetilde{u}_{j}(r)-\mu_{n}\left(T(r, u)+q n_{0}\right) \geq \frac{1}{\pi} \sum_{j=1}^{q} \widetilde{u}_{j}(r)-\mu_{n}(1+\varepsilon) T(r, u) .
$$

Finally, we get

$$
\int_{t_{n}}^{T_{n}} \frac{\sum_{j=1}^{q} \widetilde{u}_{j}(r)}{r^{\mu_{n}+1}} d r<\pi \frac{1+2 \varepsilon}{1-\varepsilon} \int_{t_{n}}^{T_{n}} \frac{r T_{-}^{\prime}(r, u)}{r^{\mu_{n}+1}} d r .
$$

Hence there is a sequence $r_{n} \in\left[t_{n}, T_{n}\right]$ such that

$$
\sum_{j=1}^{q} \widetilde{u}_{j}\left(r_{n}\right)<\pi \frac{1+2 \varepsilon}{1-\varepsilon} r_{n} T_{-}^{\prime}\left(r_{n}, u\right)
$$

for large enough $n$ 's. Since our tracts are strong,

$$
\widetilde{u}_{j}\left(r_{n}\right)=\max _{|z|=r_{n}} u_{j}(z) \geq \max _{\substack{|z|=r_{n} \\ z \in \Gamma_{j}}} u_{j}(z)=\max _{\substack{|z|=r_{n} \\ z \in \Gamma_{j}}} u(z)>(1-\varepsilon) \max _{|z|=r_{n}} u(z) .
$$

From the definition of $b(\infty, u)$ we get

$$
\widetilde{u}_{j}\left(r_{n}\right)>(1-\varepsilon)^{2} b(\infty, u) r_{n} T_{-}^{\prime}\left(r_{n}, u\right) .
$$

Hence

or

$$
q(1-\varepsilon)^{2} b(\infty, u)<\pi \frac{1+2 \varepsilon}{1-\varepsilon},
$$

$$
q<\frac{\pi}{b(\infty, u)} \cdot \frac{1+2 \varepsilon}{(1-\varepsilon)^{3}} .
$$

Since $\varepsilon>0$ is arbitrary and $q$ is an arbitrary natural number such that $q \leq p$, we have proved Theorem 2 .

\section{References}

[1] A. Baernstein, Integral means, univalent functions and circular symmetrization, Acta Math. 133 (1974), 139-169.

[2] W. Bergweiler and H. Bock, On the growth of meromorphic functions of infinite order, J. Anal. Math. 64 (1994), 327-336. 
[3] A. Eremenko, An analogue of the defect relation for the uniform metric, Complex Variables Theory Appl. 64 (1997), 83-97.

[4] M. Essen and D.F. Shea, Applications of Denjoy integral inequalities and differential inequalities to growth problems for subharmonic and meromorphic functions, Proc. Roy. Irish Acad. Sect. A 82 (1982), 201-216.

[5] W. K. Hayman, Multivalent Functions, Cambridge Univ. Press, Cambridge, 1958.

[6] -, Meromorphic Functions, Clarendon Press, Oxford, 1964.

[7] W. K. Hayman and P. B. Kennedy, Subharmonic Functions, Vol. I, Academic Press, 1976.

[8] I. I. Marchenko, On the magnitudes of deviations and spreads of meromorphic functions of finite lower order, Mat. Sb. 186 (1995), 391-408.

[9] —, On asymptotic values of entire functions, Izv. Math. 63 (1999), 549-560.

[10] I. I. Marchenko and A. Szkibiel, On strong tracts of subharmonic functions of finite lower order, Mat. Studii 22 (2004), 35-44.

[11] M. N. M. Talpur, A subharmonic analogue of Iversen's theorem, Proc. London Math. Soc. (3) 32 (1976), 181-192.

Institute of Mathematics

University of Szczecin

Wielkopolska 15

70-451 Szczecin, Poland

E-mail: marchenko@wmf.univ.szczecin.pl

olaszkibiel@poczta.onet.pl

Received 23.5.2007

and in final form 4.7.2007 